\title{
Allogeneic GM-CSF-Secreting Breast Cancer Vaccine
}

National Cancer Institute

\section{Source}

National Cancer Institute. Allogeneic GM-CSF-Secreting Breast Cancer Vaccine. NCI

Thesaurus. Code C48371.

An allogenic vaccine consisting of irradiated breast cancer cells transfected with the granulocyte macrophage-colony-stimulating factor (GM-CSF) gene. Upon vaccination, the genetically modified cells secrete GM-CSF, thereby potentiating a tumor-specific $T$ cell response against breast cancer cell-asociated antigens. 